\title{
ON DERIVATIVE AND TRANSLATIONAL BASES FOR PERIODIC FUNCTIONS ${ }^{1}$
}

\author{
R. E. EDWARDS
}

1. Introduction. It has been shown by Hartman and Wintner $[1]^{2}$ that if $f(x)=\sum_{-\infty}^{\infty} F(n) e^{i n x}$ is a regular-analytic periodic function such that $F(n) \neq 0$ for all $n$, then the functions $f(x), f^{\prime}(x)$, $f^{\prime \prime}(x), \cdots$ are fundamental in the sense of uniform convergence amongst all continuous periodic functions. As is pointed out in [1], the assumption of regular-analyticity may be replaced by a suitable form of quasi-analyticity. It is the object of this paper to exploit this extension, and to consider the related problem for translational bases.

I wish to express here my thanks to Professor J. L. B. Cooper of University College, Cardiff, for the help and advice received from him during the preparation of this paper. In addition, I am grateful to a referee for pointing out two errors in the original manuscript.

2. The space of continuous periodic functions. We denote by $E$ the Banach space of all continuous functions with period $2 \pi$, the norm on $E$ being

$$
\|f\|=\sup _{x}|f(x)| .
$$

The $n$th Fourier coefficient of $f(x) \in E$ will be denoted by $F(n)$, the corresponding capital letter indicating passage from the function to its Fourier coefficients.

It is well known that the topological dual $E^{\prime}$ of $E$ is identifiable with the set of all functions $\mu(x)$ of bounded variation over $-\pi \leqq x \leqq \pi$, the duality being expressed by the bilinear functional

$$
(f, \mu)=\int_{-\pi}^{\pi} f(-x) d \mu(x)
$$

we shall not distinguish between functions $\mu(x)$ which give rise to the same functional on $E$.

If $f \in E, P(f)$ and $D(f)$ will denote the closed vector subspaces of $E$ spanned respectively by all the functions $e^{i n x}$ with $n$ such that $F(n)$

Received by the editors August 25, 1950.

1 Most of this paper forms part of a thesis approved for the degree of doctor of philosophy in the University of London. The thesis was written under the direction of Professor J. L. B. Cooper.

${ }^{2}$ Numbers in brackets refer to the references at the end of the paper. 
$\neq 0$, and by the set of derivatives of all orders of $f$, the consideration of $D(f)$ being conditional on the existence of these derivatives. Likewise, if $A$ is any given set of real numbers (which may be assumed reduced modulo $2 \pi$ if this is convenient), $T(A, f)$ is the closed vector subspace of $E$ spanned by all the translations $f_{t}(x)=f(x+t)$ of $f$ with $t$ ranging over $A$. In case $A$ contains, modulo $2 \pi$, all real numbers, we write $T(f)$ in place of $T(A, f)$.

3. The translations of a general element of $E$. Since it is plain that we have always $T(A, f) \subset P(f)$ and $D(f) \subset P(f)$ whenever $D(f)$ is defined, the best we can hope for is that $T(A, f)$ and/or $D(f)$ shall exhaust $P(f)$. For a general element $f$ of $E, D(f)$ is not defined; and in this case we are able to prove only that $T(f)=P(f)$. Of course, this last implies that $T(A, f)=P(f)$ whenever $A$ is dense (modulo $2 \pi$ ): that this is the best possible results in general is shown in [2].

Theorem 1. For any $f \in E, T(f)=P(f)$.

Proof. If $\mu \in E^{\prime}$ is orthogonal to $T(f)$, the function of $t$ defined by

$$
M(t) \equiv\left(f_{t}, \mu\right)=\int_{-\pi}^{\pi} f(t-x) d \mu(x)=f * \mu(t)
$$

is zero for all $t$. Now $M(t)$ has the Fourier coefficients equal to

$$
F(n) \cdot \int_{-\pi}^{\pi} e^{-i n x} d \mu(x) .
$$

These must all be zero, so that

$$
\int_{-\pi}^{\pi} e^{-i n x} d \mu(x)=0
$$

whenever $F(n) \neq 0$. That is, $\mu$ is orthogonal to all functions $e^{i n x} \in P(f)$. The result now follows from the Hahn-Banach theorem.

We observe that the result could also be proved by making $E$ into a commutative Banach algebra (without unit) by defining the product $f * g$ of $f, g \in E$, to be their convolution over a period:

$$
f * g(x)=(2 \pi)^{-1} \int_{-\pi}^{\pi} f(x-y) g(y) d y .
$$

We have then only to apply a known resolution theorem for ideals in such algebras; see Segal [3, Theorem 2.2 and Corollary 2.2.1].

4. Functions of $E$ with "small" Fourier coefficients. We begin by formulating two general principles which serve to make plain the 
relevance of quasi-analyticity to the problems we are considering. For a given $f \in E$, let $E^{\prime}(f)$ be the class of all functions of the form $f * \mu, \mu$ ranging over $E^{\prime}$. We have then

(A) In order that $T(V, f)=P(f)$ shall hold for a given $f \in E$ and all nonvoid open sets $V$, it is necessary and sufficient that the class $E^{\prime}(f)$ be quasi-analytic (I) (see $[4$, p. 50]).

(B) In order that $D(f)=P(f)$ shall hold for a given $f \in E$, it is necessary and sufficient that the class $E^{\prime}(f)$ be quasi-analytic $(\Delta)$ (see $[4$, p. 130]).

Both of these principles follow from the Hahn-Banach theorem. Consider, for example, (A). If $f \in E$, if $V$ is a nonvoid open set, and if $\mu \in E^{\prime}$ is orthogonal to all the $f_{t}$ with $t \in V$, we shall have $f \mu *(t)=0$ for $t \in V$. If therefore $E^{\prime}(f)$ is quasi-analytic (I), $f * \mu(t)=0$ for all $t$. This implies, as in the proof of Theorem 1 , that $\mu$ is orthogonal to all functions $e^{i n x} \in P(f)$, and hence is orthogonal to $P(f)$ itself. At this stage, the Hahn-Banach theorem serves to show that $P(f) \subset T(V, f)$. Since the reverse relation is always satisfied, the condition is proved to be sufficient.

If, on the other hand, $E^{\prime}(f)$ is not quasi-analytic (I), there is a nonvoid open interval $V$ and $\mu \in E^{\prime}$ such that $f * \mu(t)$ is zero on $V$ and is yet not identically zero. If $t_{0}$ is a point where this function is not zero, the Hahn-Banach theorem shows that $f_{t_{0}} \notin T(V, f)$, and so that $P(f) \nsubseteq T(V, f)$. This shows that the condition is necessary, and so completes the proof of (A).

Similar reasoning establishes (B).

Before using these principles to establish specific results, it is interesting to observe a relationship between $D(f)$ and $T(A, f)$ for suitable functions $f$ and suitable sets $A$.

TheOREM 2. If $f \in E$ has derivatives of all orders, and if the set $A$ has 0 as a limiting point, then $D(f) \subset T(A, f)$.

Proof. If we use the notation of the proof of Theorem 1, the function $M(t)$ has derivatives of all orders,

$$
M^{(n)}(0)=\left(f^{(n)}, \mu\right),
$$

and $M(t)=0$ for $t \in A$. By applying Rolle's theorem successively, we see that $M^{(n)}(0)=0$ for $n=0,1,2, \cdots$, and the theorem follows from the Hahn-Banach theorem.

REMARK. There is a partial reverse of this assertion for more special functions $f$ (as one might expect on the basis of the Taylor expansion whenever this possesses the necessary uniformity of convergence). For example, if $f$ is regular-analytic (which hypothesis is equivalent 
to the assumption that $F(n)=O\left(e^{-a|n|}\right)$ for some $\left.a>0\right)$, it may be shown by use of the Hahn-Banach theorem that $T(f) \subset D(f)$. However, in this case, Theorem 3 applies to show that $D(f)=P(f)$, and hence certainly that $T(f) \subset D(f)$. Theorem 2 now goes to show that in such cases we have $T(f)=D(f)=P(f)$.

From principle (B) we obtain an extension of the result of Hartman and Wintner (see $[1$, p. 8$]$ ), namely

THEOREM 3. Suppose that $p(u)(u>0)$ is such that $u \cdot p^{\prime}(u) \uparrow+\infty$ as $u \rightarrow+\infty$, and that

$$
\int_{1}^{\infty} p(u) d u / u^{2}=+\infty .
$$

Then, if $f \in E$ is such that

$$
|F( \pm n)|<e^{-p(n)} \quad(n=0,1,2, \cdots),
$$

we have $D(f)=P(f)$.

Proof. By (B), it is enough to show that the class $E^{\prime}(f)$ is quasianalytic $(\Delta)$. However, if $\mu \in E^{\prime}$, the Fourier coefficients of $f * \mu(t)$ are those of $f$ multiplied by the Fourier-Stieltjes coefficients of $\mu$. These last are plainly bounded. That $E^{\prime}(f)$ is quasi-analytic $(\Delta)$ therefore follows from [4, Theorem II, p. 79].

REMARK. By using Theorem I, pp. 78-79, of [4], we can show that if $p(u)$ is as before, except that now

$$
\int_{1}^{\infty} p(u) d u / u^{2}<+\infty
$$

then functions $f \in E$ exist for which $D(f) \neq P(f)$.

By taking $p(u)$ equal to a suitable multiple of $u$, it results from Theorem 3 that $D(f)=P(f)$ for all regular-analytic $f \in E$ : this is the original assertion of Hartman and Wintner. Also, by Theorem 2, $T(A, f)=P(f)$ whenever $f \in E$ is regular-analytic and the set $A$ has 0 as a limiting point. But this does not make full use of the regularanalyticity of $f$. We have in fact the following theorem.

THEOREM 4. If $f \in E$ is regular-analytic, then $T(A, f)=D(f)=P(f)$ provided $A$ has at least one (finite) limit point.

Proof. On account of the remark following Theorem 2, it is enough to show that $T(A, f)=P(f)$. However, if $f$ is regular-analytic, any function of class $E^{\prime}(f)$ is the restriction to the real axis of a function regular in some horizontal strip containing that axis. If any such 
function vanishes on a set $A$ of real numbers having a finite limit point, it must vanish identically. The assertion follows from this last remark. The argument makes it plain, indeed, that any set $A$ of real numbers forming a determining set (=set of uniqueness) for the class of functions regular in this strip will serve equally well.

Strong results concerning the subspaces $T(V, f)$ follow from a more direct use of (A) (combined, of course, with deep theorems on classes quasi-analytic (I)). In particular, we may record the following theorem.

Theorem 5. If $p(u)$ is as in Theorem 3, and if $f \in E$ is such that

$$
|F( \pm n)|<e^{-p(n)}
$$

holds for all integers $n>0$ with the possible exception of a sequence $\left\{n_{i}\right\}$ such that

$$
\sum_{i} 1 / n_{i}<+\infty
$$

then $T(V, f)=P(f)$ for all nonvoid open sets $V$.

Proof. This follows from (A) combined with the theorem given in $[4, \S 61$, pp. 141-142].

So far, the quasi-analyticity of $E^{\prime}(f)$ has been ensured by assuming that the Fourier coefficients of $f$ are small at infinity. But the same result can arise from gap-conditions on the Fourier series of $f$; see [4, chaps. VII and VIII]. An extreme and simple case of this nature is discussed in [2], where it is assumed that $F(n)=0$ for all $n<0$. The result here is to the effect that $T(A, f)=P(f)$ whenever the set $A$ has positive measure.

5. A stronger mode of convergence. I owe the substance of this section to some remarks made by Professor J. L. B. Cooper. For definiteness, assume that $f \in E$ satisfies the conditions of Theorem 5 ; we then know that any $g \in P(f)$ is the uniform limit of finite linear combinations of translations $f(x+t)$ of $f$ with $t \in V$. Now suppose it known a priori that $g$ has derivatives of all orders. In this case, we strengthen the mode of convergence as follows. It is plain that for any fixed $k=0,1,2, \cdots$, the function $f^{(k)}$ also satisfies the conditions of Theorem 5 (the function $p(u)$ relevant to $f^{(k)}$ being dependent on $k$, of course), and that $g^{(k)} \in P(f)$. Hence, given $\epsilon_{k}>0$, we can find a function $f_{k}$, a finite linear combination of translations $f_{t}$ of $f$ with $t \in V$, such that

$$
\left\|f_{k}^{(k)}-g^{(k)}\right\| \leqq \epsilon_{k}
$$


By successive integration it follows that

$$
\left\|f_{k}^{(j)}-g^{(j)}\right\| \leqq(4 \pi)^{k-j} \cdot \epsilon_{k} \quad(0 \leqq j \leqq k) .
$$

If we choose the numbers $\epsilon_{k}$ so small that

$$
\lim _{\boldsymbol{k} \rightarrow \infty}(4 \pi)^{k} \cdot \epsilon_{k}=0
$$

it results that, for any fixed $j$,

$$
\lim _{k \rightarrow \infty} f_{k}^{(j)}(x)=g^{(j)}(x),
$$

uniformly in $x$. That is, the convergence of $f_{k}$ to $g$ is such that the limiting process remains valid after any number of differentiations.

Similar remarks apply to Theorem 3.

6. Free systems of translations. We consider here a problem complementary to that which precedes, namely that of determining free systems of translations. The corresponding problem concerning derivatives is not trivial for a general element of $E$. Nevertheless, there is considerable similarity between the two questions, and we shall for brevity confine our remarks to translations. In connection with derivatives let us remark only that if (for example) $f \in E$ is regularanalytic, then its derivatives never form a free system (see the definition made in the next paragraph) in $E$; in fact, the first $j$ derivatives of $f$ always belong to the closed vector subspace of $E$ spanned by the remaining ones. Using the Hahn-Banach theorem, this follows from the fact that no function of class $E^{\prime}(f)$ can be a polynomial without being identically constant. This shows, incidentally, that for such $f \in E, D(f)$ is unaltered if in its definition we omit any finite number of derivatives of $f$. Results of this type have a direct analogue for translations in assertions of the calibre of Theorem 6 below.

In an arbitrary topological vector space $E$, a subset $F$ of $E$ is said to be free if, whenever $f \in F, f$ does not belong to the closed vector subspace of $E$ spanned by the subset $F-\{f\}$.

In turning to translations, the problem we have in mind has meaning relative to any translation-invariant topological vector space of functions defined on an abelian topological group. In marked contrast to the problem of fundamentality of translations in such a space, which has received a great deal of attention, the question of free systems of translations appears not to have been discussed at all. It would appear, however, that the case in which the underlying group is compact is by far the simplest: this is true of the case with which 
we are concerned in this paper, the underlying group being the factor group of the reals modulo $2 \pi$.

Given a function $f \in E$ (where $E$ may have the general significance explained in the last paragraph), we shall say that the translations of $f$ are free (or, at length, that they form a free system in $E$ ) if, for any discrete subset ${ }^{3} A$ of the underlying group, the system of translations $f_{t}$ of $f$ with $t \in A$ is free in $E$. It need hardly be remarked that, in dealing with the space $E$ defined in $\$ 2$, the topology in question is the quotient modulo $2 \pi$ of the usual metric topology on the real axis.

Returning now to the special problem in hand, it is quite obvious that a function $f \in E$ whose Fourier coefficients are too small at infinity cannot possibly have free translations; further, the vanishing of too many Fourier coefficients of $f$ can also preclude the possibility that $f$ has free translations. In both these cases, the reason why $f$ fails to have its translations free is because the class $E^{\prime}(f)$ is quasi-analytic. The question as to how many of the Fourier coefficients of $f$ may vanish and yet $f$ have its translations free remains open. Theorem 6 below is designed to show how small the Fourier coefficients of $f$ may be (at infinity) and yet $f$ have a free system of translations, it being granted that $F(n) \neq 0$ for all $n$. The conclusion of Theorem 6 is in fact stronger than the assertion that the translations of $f$ are free.

One further notion is required prior to the statement of Theorem 6. A sequence $\{A(s)\}(s=0,1,2, \cdots)$ of positive numbers is said to be of Carleman type if, when we define

$$
B(s)=(A(s))^{1 / s}, \quad \bar{B}(s)=\inf _{t \geqq s} B(t),
$$

we have

$$
\sum_{s} 1 / \bar{B}(s)<+\infty \text {. }
$$

Theorem 6. Suppose that $f \in E$ and that $F(n) \neq 0$ for all $n$. Suppose also that there is a sequence $\{A(s)\}$ of Carleman type, and a sequence $\left\{s_{n}\right\}(n=0,1,2, \cdots)$ of non-negative integers, such that for every $X>0$

$$
\sum_{-\infty}^{\infty}|F(n)|^{-1} \cdot A\left(s_{|n|}\right)(X /|n|)^{s|n|}<+\infty
$$

then $f$ has free translations in $E$.

Proof. As mentioned above, we aim to prove a little more, namely

${ }^{3}$ By this we mean that $A \cap A^{\prime}$ is void, that is, that the induced topology on $A$ is discrete. This does not, of course, mean that $A$ can have no limit point in the group. 
that $f$ has the following property.

(P) Given a set $A$, closed in the quotient topology, and a point $t_{0}$ not in $A$, the function $f_{t_{0}}$ is not in $T(A, f)$.

We begin by converting $(\mathrm{P})$ into an equivalent assertion concerning the class $E^{\prime}(f)$ :

$\left(\mathrm{P}^{\prime}\right)$ Given any neighbourhood $V$ of zero (in the quotient topology), there is a function of class $E^{\prime}(f)$ which has the value 1 at zero and is zero outside $V$ (modulo $2 \pi$ ).

To verify the equivalence of $(\mathrm{P})$ and $\left(\mathrm{P}^{\prime}\right)$, note first that there is no loss of generality involved in assuming that the arbitrarily given point $t_{0}$ is $t_{0}=0$. And, by the Hahn-Banach theorem, to require that $f \notin T(A, f)$ for any set $A$, closed in the quotient topology and not containing 0 , is the same as to require that a functional $\mu \in E^{\prime}$ exists corresponding to any given neighbourhood $V$ of zero which is orthogonal to all the $f_{t}$ with $t$ outside $V$ and which is yet not orthogonal to $f$ itself. Any such $\mu$, when multiplied by a suitable scalar, yields a function $f * \mu \in E^{\prime}$ satisfying $\left(\mathrm{P}^{\prime}\right)$.

The remainder of the proof is concerned with proving the existence of a function of class $E^{\prime}(f)$ satisfying $\left(\mathrm{P}^{\prime}\right)$. By [4, chap. V, §33], there is a not-identically-zero, infinitely differentiable function $\phi(t)$ $(-\pi \leqq t \leqq \pi)$ such that

$$
\phi^{(s)}(-\pi)=\phi^{(s)}(\pi)=0 \quad(s=0,1,2, \cdots)
$$

and

$$
\sup _{t}\left|\phi^{(s)}(t)\right|<A(s) \quad(s=0,1,2, \cdots) .
$$

Suppose that $\tau,-\pi<\tau<\pi$, is chosen so that $\phi(\tau) \neq 0$. Let $\phi(t)$ be defined everywhere by periodicity. Choose $X>0$ so large that $(-2 \pi / X$, $2 \pi / X) \subset V, V$ being the neighbourhood of zero figuring in condition $\left(\mathrm{P}^{\prime}\right)$ (assumed reduced modulo $2 \pi$ ). Put $\theta(t)=\phi(X t+\tau)$; then $\theta(t)$ is infinitely differentiable, and

$$
\begin{aligned}
\theta(0) & \neq 0, \\
\theta^{(s)}(-a) & =\theta^{(s)}(b)=0 \quad(s=0,1,2, \cdots),
\end{aligned}
$$

where

$$
-a=(-\pi-\tau) / X<0, \quad b=(\pi-\tau) / X>0 ;
$$

further

$$
\sup _{t}\left|\theta^{(s)}(t)\right|<X^{s} \cdot A(s) \quad(s=0,1,2, \cdots) .
$$

We may assume $X$ so large that $-\pi<-a<0<b<\pi$. Finally, put 


$$
\delta(t)= \begin{cases}\theta(t) & \text { for }-a \leqq t \leqq b, \\ 0 & \text { elsewhere on }-\pi \leqq t \leqq \pi\end{cases}
$$

and define $\delta(t)$ outside $[-\pi, \pi]$ by periodicity. The Fourier coefficient of $\delta(t)$ are then

$$
\Delta(n)=(2 \pi)^{-1} \int_{-a}^{b} \theta(t) e^{-i n t} d t,
$$

whence, integrating by parts $s_{|n|}$ times and using (6.3), we have

$$
|\Delta(n)|<K \cdot A\left(s_{|n|}\right) \cdot(X /|n|)^{s|n|},
$$

$K$ being an absolute constant.

If we assume the hypothesis of the theorem, (6.5) shows that the formulae

$$
M(x)=(2 \pi)^{-1} \sum_{-\infty}^{\infty}\{F(n)\}^{-1} \cdot \Delta(n) \cdot e^{i n x} \quad(-\pi \leqq x \leqq \pi)
$$

and

$$
d \mu(x)=M(x) d x
$$

are effective in defining a measure $\mu \in E^{\prime}$ such that

$$
f * \mu(t)=\delta(t)
$$

identically in $t$. By (6.2) and (6.4) it follows that the measure $\mu$ is orthogonal to $f_{t}$ when $t$ is outside $V$ (modulo $2 \pi$ ) and yet is not orthogonal to $f$ itself. The proof is therefore complete.

REMARKS.

(i) We can take, for example, $A(s)=s^{p s}$ where $1<p<+\infty$, and $s_{n}$ equal to the integral part of $n^{q}$ for $n>0$ where $0<q<1 / p$; our condition is then that

$$
\sum_{-\infty}^{\infty}|F(n)|^{-1} \cdot(X /|n|)^{(1-p q)|n|^{q}}<+\infty \quad(\text { all } X>0) .
$$

(ii) All that is required in the above proof is that the numbers

$$
c_{n}=\{F(n)\}^{-1} \cdot A\left(s_{|n|}\right)(X /|n|)^{s|n|}
$$

be the Fourier-Stieltjes coefficients of a bounded measure $\mu \in E^{\prime}$ (for each $X>0$ ). But this itself implies that the $c_{n}$ are at any rate bounded, and, by replacing them by $c_{n} /\left(1+|n|^{2}\right)$ (say), our hypothesis will be satisfied. But remark (i) shows that factors of the type $\left(1+|n|^{2}\right)^{-1}$ are of no importance compared with the remaining terms. 
Added in proof. The questions outlined at the beginning of $\$ 6$, namely those concerning the freedom or independence of translations, are discussed at greater length and in more generality in a paper to appear in the Annales de l'Institut Fourier. The restriction that $F(n) \neq 0$ for all $n$ is there removed.

\section{REFERENCES}

1. P. Hartman and A. Wintner, Derivative bases, Revista Universidad del Tucumán. Ser. A vol. 7 (1949).

2. R. E. Edwards, $A$ property of the class of functions regular in the unit circle and $a$ theorem on translations, J. London Math. Soc. vol. 25 (1950).

3. I. E. Segal, The group algebra of a locally compact group, Trans. Amer. Math. Soc. vol. 61 (1947).

4. S. Mandelbrojt, Séries de Fourier et classes quasi-analytiques de fonctions, Paris, Borel Collection, 1935.

Birkbeck College, University of London 